\title{
RETINA LAYER SEGMENTATION AND SPATIAL ALIGNMENT OF ANTIBODY EXPRESSION LEVELS
}

\author{
Nhat Vu, Pratim Ghosh, and B. S. Manjunath \\ Center for Bio-informatics \\ Department of Electrical and Computer Engineering \\ University of California, Santa Barbara, CA 93106-9560 \\ http://www.bioimage.ucsb.edu/
}

\begin{abstract}
The expression levels of rod opsin and glial fibrillary acidic protein (GFAP) capture important structural changes in the retina during injury and recovery. Quantitatively measuring these expression levels in confocal micrographs requires identifying the retinal layer boundaries and spatially corresponding the layers across different images. In this paper, a method to segment the retinal layers using a parametric active contour model is presented. Then spatially aligned expression levels across different images are determined by thresholding the solution to a Dirichlet boundary value problem. Our analysis provides quantitative metrics of retinal restructuring that are needed for improving retinal therapies after injury.
\end{abstract}

Index Terms - Active contours, layer segmentation

\section{INTRODUCTION}

Recent advances in cytochemical antibody labeling and confocal imaging have allowed biologists to observe protein expressions in relatively small tissue sections with greater detail. In studying the mammalian retina and its response to injury, antibody labeling can target specific tissue layers or cell populations and reveal intricate structural changes that are closely correlated with functional impairments.

In retinal detachment experiments (separating the neural retina from the pigmented epithelium, Fig. 1), two important antibody labels are used to target highly responsive proteins. The first, rod opsin, is found in rod photoreceptor outer segments under normal conditions and is a good indicator of the rod's ability to detect light stimuli. The second, glial fibrillary acidic protein (GFAP), is predominantly localized in Muller cell endfoot regions under normal conditions (Fig. 2(a)). These Muller cells have been found to be highly reactive to detachment, undergoing hypertrophy and triggering a cascade of undesirable events leading to decreased neuronal stability and potentially significant vision impairment [1].

This work is supported by NSF ITR grant \#ITR-0331697.

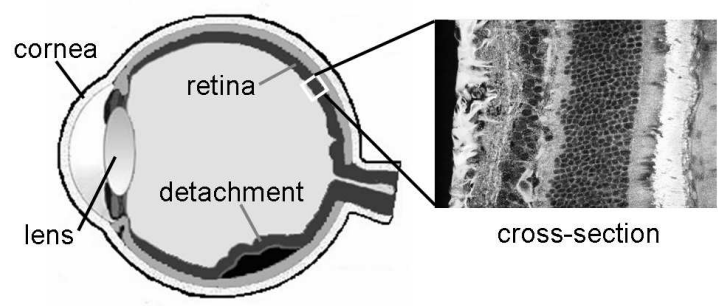

Fig. 1. Retinal detachment.

To quantify the extent of tissue restructuring during detachment, both the changes in magnitude and location of the antibody expression levels are important. In this paper, methods to detect retinal boundaries and to spatially correspond antibody expression levels across different images are presented. The analysis is summarized in the following steps: 1) For each image, the relevant retinal boundaries are detected using a modified parametric active contour model previously presented in [2]. 2) The retinal layers are divided along their lengths into smaller corresponding sub-layers using the solution of the Laplace equation with Dirichlet boundary conditions. 3) The expression level of each sub-layer are computed, and comparisons are made for retinas at different experimental stages. To the best of our knowledge, there has been no previous quantitative analysis of retinal protein expression levels with spatial correspondence.

\subsection{Retina Image Dataset}

The dataset is composed of images of rod opsin (red channel) and GFAP (green channel) labeled feline retina cross-sections during four stages of retinal detachment. There are 28, 36, 13, and 45 images of normal or undetached, 3-day, 7-day, and 28day detached retinas, respectively. Because imaging requires destroying the tissue samples and the specimens are costly, only the most interesting stages are fully explored, leading to an unequal number of images in each stage. All images are $512 \times 768$ pixels in size. Fig. 2 shows examples of images from each stage of retinal detachment. 


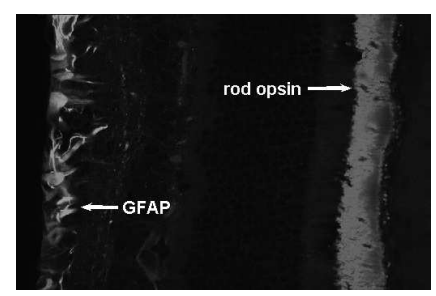

(a) normal

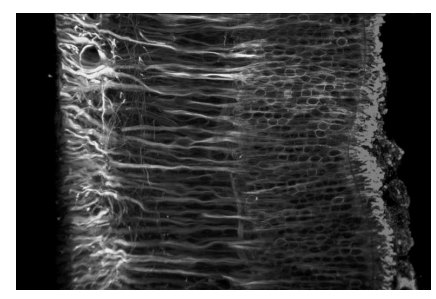

(c) 7-day

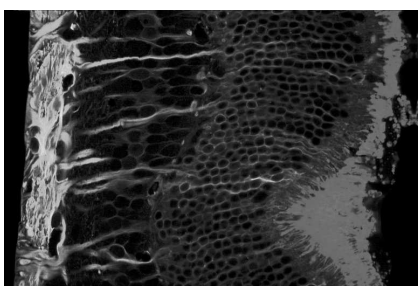

(b) 3-day

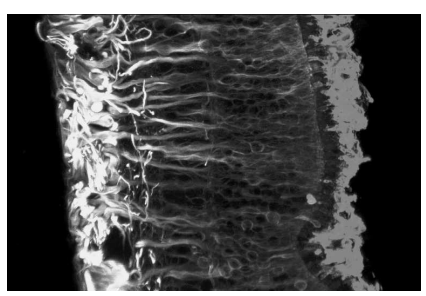

(d) 28-day
Fig. 2. Rod opsin and GFAP labeled images for each stage of retinal detachment.

\section{LAYER SEGMENTATION}

The discrete dynamic contour model of Lobregt and Viergever [3] is used to detect the retinal layer boundaries. The parametric framework is preferred because it easily allows for simple open contours, which is the case with retinal layer boundaries. Furthermore, a contiguous retinal layer may contain irregular patterns that would cause geometric methods to divide the layer into several isolated blobs. Although there are numerous other parametric active contour models that are likely to perform equally well (see [4] and the references therein for an overview), the most important aspect of using these models is defining the external or data-driven force.

\subsection{External Force}

The external force formulation is based on the perpendicular intensity profile matching framework proposed in [2], with some important modifications. Consider a discrete contour composed of $N$ vertices at positions $\left\{\mathbf{v}_{i} \mid i=1 \ldots N\right\}$. At vertex $i$, construct a perpendicular intensity profile $P_{i}$ consisting of $M$ samples spaced at $d s$ pixels apart such that

$$
P_{i}(j)=I\left(\mathbf{v}_{i}+j \cdot d s \cdot \hat{\mathbf{n}}_{i}\right) \text { for }-m \leq j \leq m,
$$

where $I$ is the intensity image, $\hat{\mathbf{n}}_{i}$ is the unit normal at $\mathbf{v}_{i}$, and $m=(M-1) / 2$ (Fig. 3). The external force at $\mathbf{v}_{i}$ is calculated by matching the intensity profile $P_{i}$ with a target profile $P_{i}^{t}$. The matching is done by defining an external energy $E_{i}$ as follows:

$$
E_{i}(j)=w(j) \sum_{k=-m}^{m}\left|P_{i}^{t}(k)-P_{i}(j+k)\right|,
$$

where $w(j)=M /(M-2|j|)$ and $-m \leq j \leq m$. The energy $E_{i}$ can be considered as the normalized cumulative absolute gray value differences [2], with $w(j)$ normalizing the sum to the proper number of profile samples involved in the summation. The external force is then defined as

$$
f_{e x t}^{i}=-\frac{\partial E_{i}}{\partial \hat{\mathbf{n}}_{i}}\left(\mathbf{v}_{i}\right) .
$$

This external force deforms the contour so that each vertex converges toward a local minimum in the external energy distribution.

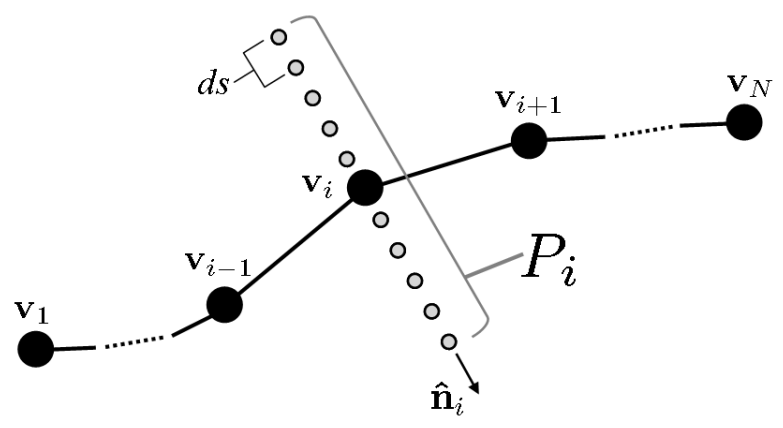

Fig. 3. Perpendicular profile $P_{i}$ at vertex $\mathbf{v}_{i}$.

\subsection{Target Profile and Training}

In [2], the active contour is used to track moving boundaries in an image sequence. The final profiles $P_{i}$ 's of the converged contour from the previous image are used as the target profiles $P_{i}^{t}$ 's in the current image. However, the segmentation of retinal layers is performed on static images, requiring a different definition for the target profiles $P_{i}^{t}$ 's. Consequently, a training stage is employed to obtain the target profiles.

Given a set of images under similar experimental conditions, one image is selected, and the desired boundaries are manually traced. Then at sample points along each boundary, the perpendicular intensity profiles are computed and averaged to obtain a single target profile $P^{t}$ for that boundary. Hence, instead of having a different target profile for each vertex, all vertices share the same target profile $P^{t}$.

\subsection{Modified External Force}

Retinal detachment often causes large deformation in the layers, and thus obtaining good initializations for each boundary is a challenging task. To make the algorithm more insensitive to initialization, the lengths of $P^{t}$ and $P_{i}$ should be large so that an initialization far away from the target boundary will still converge correctly. A long $P^{t}$, however, will span across neighboring layer boundaries, which can have a significant effect on the profile matching energy $E_{i}$.

To limit the contributions from the neighboring boundaries, a windowing term $\alpha(k)$ is incorporated within the external energy definition (2). The modified energy is given by

$$
E_{i}(j)=w(j) \sum_{k=-m}^{m} \alpha(k)\left|P^{t}(k)-P_{i}(j+k)\right|,
$$


where $\alpha(k)=\exp \left(-k^{2} / \sigma^{2}\right)$. The parameter $\sigma$ is set to be approximately smaller than the minimum distance between any two neighboring borders in the data set, which significantly reduces the undesirable contributions from neighbors to the overall profile matching energy.

\subsection{Parameter Settings, Initialization, and Results}

The algorithm is trained to detect the five boundaries separating: 1) the background and the ganglion cell layer (bg/GCL), 2) the GCL and the inner nuclear layer (GCL/INL), 3) the INL and outer nuclear layer (INL/ONL), 4) the ONL and the rod outer segment (ONL/OS), and 5) the OS and background (OS/bg) (Fig. 4). Although the retina can be further divided into more layers, retinal detachment causes cells in these layers to migrate, making the layer borders difficult even for a trained biologist to differentiate.

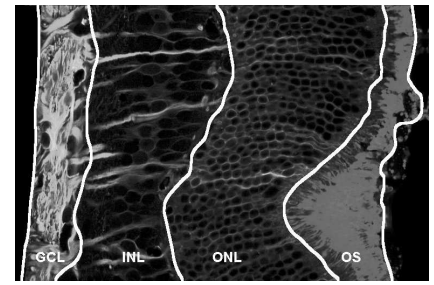

(a) 3-day

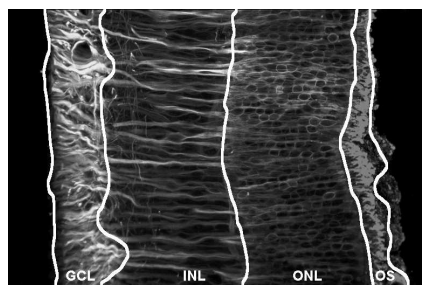

(b) 7-day
Fig. 4. Segmentation results for images in Fig. 2 (b) and (c).

The intensity from the histogram-equalized rod opsin and GFAP channels are used to compute the external force. For each vertex $\mathbf{v}_{i}$, two forces $f_{\text {rod }}^{i}$ and $f_{G F A P}^{i}$ are computed according to (3) and combined (equally weighted sum) to form the external force $f_{\text {ext }}^{i}$. Because the magnitudes of $f_{\text {rod }}^{i}$ and $f_{G F A P}^{i}$ are proportionally dependent on the amount of protein signal (intensity) present at each layer, a more sophisticated weighting scheme is not necessary.

The perpendicular profile parameters are set to $M=65$ and $d s=5$. These values ensure a long enough profile $P_{i}$ so that the algorithm is less sensitive to initialization. The window parameter $\sigma$ is set to 30 pixels (see section 2.3). The contour vertices were kept at approximately 15 pixels apart, but this spacing, along with the choice of $d s$, is a matter of computational efficiency. We have observed that with reasonable initializations, these parameters can vary through a wide range of values without noticeably affecting the results.

For initialization, a prior knowledge about the relative ordering of the retinal layers is incorporated. The two boundaries bg/GCL and OS/bg are found by initializing two contours near the left and right edges of the image, respectively. Next, the INL/ONL contour is initialized halfway between the previous two borders. Then, the GCL/INL contour is initialized at $10 \%$ of the distance from the bg/GCL to the INL/ONL boundary. Finally, the ONL/OS contour is initialized at $10 \%$ of the distance from the OS/bg to the INL/ONL boundary. Due to space limitations, only two results are shown in Fig. 4.

\subsection{Validation}

The segmentation error is found by comparing the computed boundaries with those obtain manually, i.e. with the ground truth. Often point correspondences are needed in order to measure the position error between the two discrete contours. However in this validation, the position error is defined as the thickness of the region(s) between the computed and ground truth boundaries. Using the Eulerian PDE approach proposed by Yezzi and Prince [5], the thickness between the computed and ground truth boundaries is found without explicitly having to find point correspondences between them.

Table 1. Layer segmentation error (mean \pm std pixels).

\begin{tabular}{|l||l|l|l|l|}
\hline & Normal & 3-day & 7-day & 28-day \\
\hline \hline bg/GCL & $2.2 \pm 2.1$ & $5.2 \pm 4.7$ & $2.1 \pm 2.2$ & $3.8 \pm 3.6$ \\
\hline GCL/INL & $10.9 \pm 10.9$ & $26.9 \pm 19.6$ & $19.3 \pm 15.6$ & $22.5 \pm 20$ \\
\hline INL/ONL & $5.4 \pm 3.9$ & $5.2 \pm 6.1$ & $5.0 \pm 5.9$ & $7.3 \pm 7.4$ \\
\hline ONL/OS & $7.5 \pm 9.6$ & $7.0 \pm 7.3$ & $9.9 \pm 11.8$ & $6.9 \pm 6.5$ \\
\hline OS/bg & $5.0 \pm 3.9$ & $4.4 \pm 4.4$ & $9.9 \pm 8.4$ & $7.4 \pm 6.3$ \\
\hline
\end{tabular}

The validation is performed using images from the same stage of retinal detachment. For each run, the process selects one image for training, tests the algorithm on the rest of the images, and computes the errors for that run. The process repeats, sequentially selecting a new training image each time and testing on the rest, until all images have been selected once. Table 1 summarizes the average and standard deviation error of all the segmentation results with the ground truth. As is expected, the errors increase for the latter stages of detachment due to pronounced physical changes in the retina. The GCL/INL boundary is visually the most ambiguous and has the largest errors. To provide some perspective, the average thicknesses of the GCL, INL, ONL, and OS for 7-day detached samples are 98.9, 224.3, 232.6, and 78.0 pixels, respectively, and hence the errors are deemed acceptable.

\section{EXPRESSION LEVEL CORRESPONDENCE}

Measures such as the percentage of GFAP penetration into the ONL or the change in rod opsin labeling across the ONL are important biologically and need the spatial correspondence of the ONLs throughout the image set. Here, spatial correspondence is achieved by dividing each layer into thinner sub-layers along the layer length (Fig. 5) and making ordered, one-to-one correspondences among the sub-layers from different images.

For each layer $R$ with two boundaries $\partial R_{0}$ and $\partial R_{1}$, the solution $u$ to the Laplace equation

$$
\Delta u=0, \text { with } u\left(\partial R_{0}\right)=0 \text { and } u\left(\partial R_{1}\right)=1,
$$

provides a set of equal potential contours between the two boundaries. The layer $R$ is sliced along its length by thresholding $u$ at values between 0 and 1 . If $L$ sub-layers are needed, 


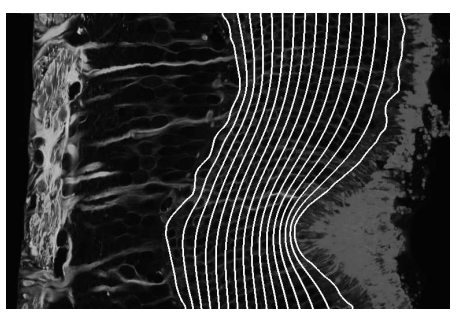

Fig. 5. ONL sliced into 15 sub-layers.

then each sub-layer $l_{n}$ is given by

$$
l_{n}=\{u \mid(n-1) \Delta u \leq u<n \Delta u\},
$$

where $\Delta u=1 / L$ and $n=1,2, \ldots L$. Fig. 5 shows the result of slicing the ONL layer into 15 sub-layers. After slicing, the protein expression statistics, such as mean and standard deviation, for each sub-layer can be easily computed.

\section{PRELIMINARY BIOLOGICAL ANALYSIS}

After segmentation, the GCL, INL, ONL, and OS layers in all images are sliced into 8, 20, 20 and 10 sub-layers, respectively. For each detachment stage, the average rod opsin and GFAP expression levels in each sub-layers are computed and plotted in Fig. 6. Note that the $\mathrm{x}$-axis identifies the relative locations in the retina where the expression values were computed. There are two interesting trends in the figure. First, the rod opsin level in the ONL increased shortly after detachment, but after 28 days of detachment, this level decreased toward the normal level. This may suggest that the rod cells are recovering some of their normal functions without any intervention such as reattachment. The second trend shows the GFAP level in the INL and ONL increased throughout detachment and remain high even after 28 days. This may suggest that once the Muller cells undergo cytoskeletal changes, the effects are difficult to reverse. Student t-tests with $p=0.05$ confirmed that the differences in expression levels of these two trends are significant.

\section{CONCLUSION}

In this paper, a method to analyze the expression levels of antibody labeling in retinal confocal images is presented. First, the retinal layer boundaries are detected using a modified parametric active contour framework adapted from [2]. Then each retinal layer is sliced into thinner sub-layers by thresholding the solution to Laplace's equation with Dirichlet boundary conditions. The slicing of the layers allows for the spatial alignment of expression levels. Comparison of expression levels from the four stages of retinal detachment corroborates prior qualitative observations [1] and may lead to improvments in retinal reattachment therapies. Future work includes preprocessing the image using texture classification
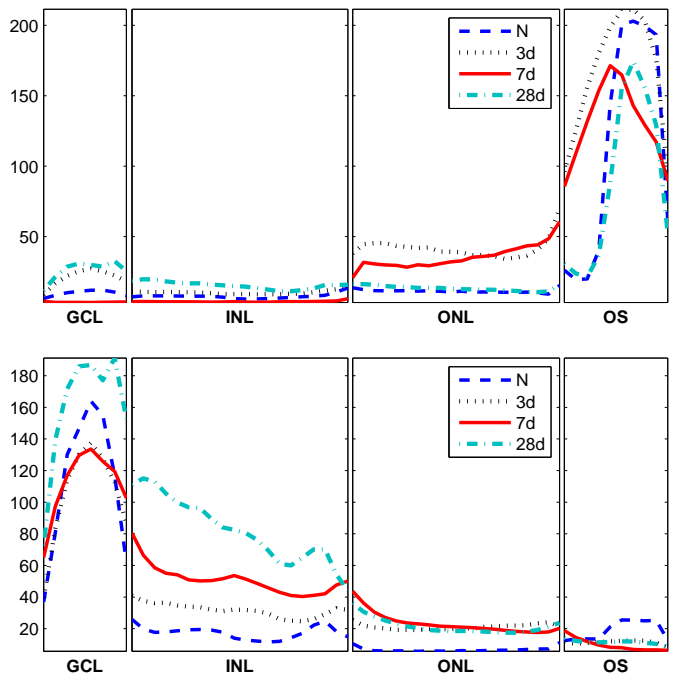

Fig. 6. Mean antibody expression levels (top: rod opsin, bottom: GFAP)

and incorporating more antibody channels to improve boundary detection, as well as more in depth analysis of biological trends.

\section{ACKNOWLEDGMENTS}

We would like to thank Dr. Geoffrey Lewis and Prof. Steven Fisher from the Neuroscience Research Institute, UCSB for the retina image dataset and for their helpful discussions.

\section{REFERENCES}

[1] S. K. Fisher, G. P. Lewis, K. A. Linberg, and M. R. Verardo, "Cellular remodeling in mammalian retina: results from studies of experimental retinal detachment," Progress in Retinal and Eye Research, vol. 24, pp. 395431, 2005.

[2] G. Hautvast, S. Lobregt, M. Breeuwer, and F. Gerritsen, "Automatic contour propagation in cine cardiac magnetic resonance images," IEEE Trans. Med. Imag., vol. 25, no. 11, pp. 1472-1482, Nov. 2006.

[3] S. Lobregt and M. A. Viergever, "A discrete dynamic contour model," IEEE Trans. Med. Imag., vol. 14, no. 1, pp. 12-24, Mar. 1995.

[4] C. Xu and J. L. Prince, "Snakes, shapes, and gradient vector flow," IEEE Trans. Image Processing, vol. 7, no. 3, pp. 359-369, Mar. 1998.

[5] J. Yezzi, A. J. and J. L. Prince, "An eulerian PDE approach for computing tissue thickness," IEEE Trans. Med. Imag., vol. 22, no. 10, pp. 1332-1339, Oct. 2003. 